\title{
WikiPathways App for Cytoscape: Making biological pathways amenable to network analysis and visualization [version 1;
}

\section{peer review: 2 approved]}

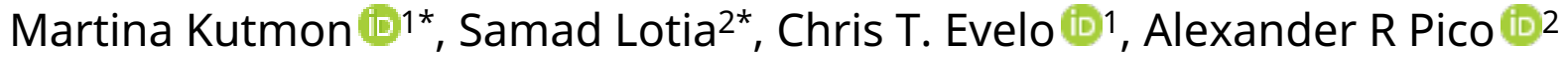 \\ ${ }^{1}$ Department of Bioinformatics - BiGCaT, Maastricht University, Maastricht, 6229 ER, The Netherlands \\ ${ }^{2}$ Gladstone Institutes, San Francisco, CA 94158-2261, USA \\ * Equal contributors
}

V1 First published: 01 Jul 2014, 3:152

https://doi.org/10.12688/f1000research.4254.1

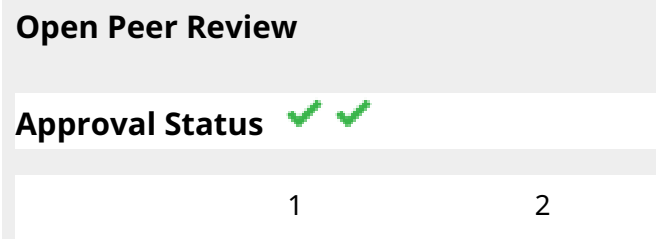

Abstract

In this paper we present the open-source WikiPathways app for Cytoscape (http://apps.cytoscape.org/apps/wikipathways) that can be used to import biological pathways for data visualization and network analysis. WikiPathways is an open, collaborative biological pathway database that provides fully annotated pathway diagrams for manual download or through web services. The WikiPathways app allows users to load pathways in two different views: as an annotated pathway ideal for data visualization and as a simple network to perform computational analysis. An example pathway and dataset are used to demonstrate the functionality of the WikiPathways app and how they can be combined and used together with other apps. More than 2000 downloads between its first release in August 2013 and the submission of the paper in May 2014 highlight the importance and adoption of the app in the network biology field.

\section{version 2}

(revision)

11 Sep 2014

version 1

01 Jul 2014

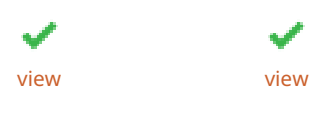

1. Christian A Grove, California Institute of Technology, Pasadena, CA, USA

2. Ted Goldstein, University of California Santa Cruz, Santa Cruz, CA, USA

Any reports and responses or comments on the article can be found at the end of the article.

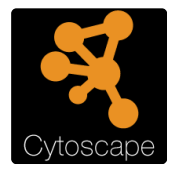

This article is included in the Cytoscape gateway. 
Corresponding authors: Martina Kutmon (martina.kutmon@maastrichtuniversity.nl), Samad Lotia (samad.lotia@gladstone.ucsf.edu)

Competing interests: No competing interests were disclosed.

Grant information: We would like to acknowledge funding from NIGMS R01-GM100039 and NIDA U54-DA036134 (ARP, SL). This work was (co)financed by the Netherlands Consortium for Systems Biology (NCSB) which is part of the Netherlands Genomics Initiative/Netherlands Organisation for Scientific Research (MK, CTE).

The funders had no role in study design, data collection and analysis, decision to publish, or preparation of the manuscript.

Copyright: ( $) 2014$ Kutmon $\mathrm{M}$ et al. This is an open access article distributed under the terms of the Creative Commons Attribution License, which permits unrestricted use, distribution, and reproduction in any medium, provided the original work is properly cited. Data associated with the article are available under the terms of the Creative Commons Zero "No rights reserved" data waiver (CC0 1.0 Public domain dedication).

How to cite this article: Kutmon M, Lotia S, Evelo CT and Pico AR. WikiPathways App for Cytoscape: Making biological pathways amenable to network analysis and visualization [version 1; peer review: 2 approved] F1000Research 2014, 3:152

https://doi.org/10.12688/f1000research.4254.1

First published: 01 Jul 2014, 3:152 https://doi.org/10.12688/f1000research.4254.1 


\section{Introduction}

Pathways are commonly used as models for understanding biological processes. WikiPathways ${ }^{1}$ is an open, collaborative, wiki-based website for the curation of biological pathways that are more than just images. WikiPathways provides easy-to-use drawing and annotation tools to capture identities, relationships, comments and literature references for each pathway element and interaction. Contributed pathways are displayed like articles at WikiPathways and can be downloaded manually or programmatically through web services. This opens the possibility for pathway information to be accessed by other software tools for data visualization, computational analysis and the interpretation of large-scale experimental data.

Utilizing WikiPathways web services, we developed an app for Cytoscape $^{2}$, a network visualization and analysis software platform. The app queries and imports pathways from WikiPathways within the Cytoscape environment. Cytoscape's core concepts are networks (nodes and edges), tables (rows and columns) and styles, which map table values to the visual properties of networks. Cytoscape leverages a rich ecosystem of apps to provide additional domainspecific semantics and data types, as well as custom visualization and analysis capabilities. With the WikiPathways app, we implemented two ways to represent a pathway as a Cytoscape network. In the first way, pathways are loaded with the complete visual appearance of the original at WikiPathways, including graphical annotations and labels. Once in Cytoscape, experimental data can be loaded as tables and visually mapped onto these pathway-style networks to provide biological context. In the second way, pathways are loaded as simplified networks, focusing on the biological entities and their interactions without any of the graphical elements of the original pathway diagram. The basic network style is ideal for topological analyses, network merging and automatic layout.

In this paper we present the implementation and usage of the WikiPathways app for Cytoscape. By bringing pathways into Cytoscape using the WikiPathways app, it is possible to make full use of pathway models with custom visualizations and computational analyses.

\section{Implementation}

The WikiPathways app was developed for Cytoscape 3, which introduced a completely new software architecture. The new architecture is built on top of Open Service Gateway Initiative (OSGi) ${ }^{3}$, a software framework of pluggable modules and services. To be able to take advantage of the new architecture (Cytoscape API version 3.0.0), the predecessor to the WikiPathways app, the GPML Plugin, had to be rewritten.

\section{Pathway import}

The WikiPathways app employs the new architecture of Cytoscape in two ways. First, the app exports a user interface that can query and import pathways from the WikiPathways web service. Thanks to the service architecture in Cytoscape, this interface is seamlessly incorporated into Cytoscape's "Import from Public Databases" dialog. Second, the app provides an API for programmatic access to the WikiPathways web services and the GPML file importer. Other apps can use the API to make queries to the WikiPathways web services and import GPML files without having to bundle the WikiPathways app. When the WikiPathways app is loaded in Cytoscape, the app registers the implementation of its API with the OSGi module system. Other apps can then request the API implementation through OSGi.

\section{Visualization}

The new architecture also posed new challenges that required us to innovate with respect to visual styles. The new architecture includes a revamped model to represent networks. This model decouples the network topology and table data from its visual style. Visual styles constitute Cytoscape's view model. When a node or edge is created in the network model, its view object is only created after a triggering of an event. Cytoscape does this to avoid redrawing of the network canvas while an app is still in process of building the network. Indeed, as the WikiPathways app reads a GPML file, it creates a series of nodes and edges in a network to represent the pathway. During this process, the app needs to assign visual styles to the nodes and edges it creates. However, as new nodes and edges are being added to the network, their view objects do not exist yet, making it impossible to assign their visual styles. To address this issue, we created a class called DelayedVizProp that stores our desired visual styles for nodes and edges. Once the network has been fully built, the app tells Cytoscape to create the view objects for the new nodes and edges. After that, the app looks through the DelayedVizProp instances and assigns nodes and edges their desired visual style.

\section{Dependencies}

The app relies on the PathVisio core library ${ }^{4}$ to read GPML files. The PathVisio library is included in the app. In previous versions of Cytoscape, apps that included libraries often conflicted with each other. Users had to painstakingly uninstall conflicting apps for Cytoscape to become usable again. OSGi solves this problem by insulating Cytoscape modules and apps from each other. Due to OSGi's architecture in Cytoscape 3, the integrated PathVisio library is hidden from other apps and modules in Cytoscape and cannot conflict with them.

The app also uses the Apache HTTP Client library to make HTTP requests to the WikiPathways REST server. We avoided the Java built-in HTTP client class (java.net.HttpURLConnection), which is used frequently in Cytoscape and other apps. This class does not support cancellation. Proper cancellation is important for a responsive user interface. Users behind an interrupted internet connection should be able to back out of a WikiPathways request and return to Cytoscape. Each HTTP request is wrapped in a task, a unit of work in Cytoscape. When the user clicks cancel during the task execution, the app terminates the underlying HTTP request by calling the abort method in the Apache HTTP Client library.

\section{Results}

The WikiPathways app in Cytoscape provides convenient access to the community-curated collection of biological pathways at WikiPathways. The functionality of the app is demonstrated here using the human Cardiac Hypertrophic Response pathway from WikiPathways (http://wikipathways.org/instance/WP2795) combined with an unpublished RNA-seq dataset that reflects gene expression levels during differentiation of cardiac stem cells (see Dataset 1). The $\log \mathrm{FC}$ from timepoint 6 hrs vs control is visualized on the pathway. The human Cardiac Hypertrophic Response pathway 
contains gene products and metabolites involved in the intracellular signal-transduction pathways that coordinate Cardiac Hypertrophic Response. As described above, the WikiPathways app allows users to load pathways in two different views, as an annotated pathway and as a simple network (see Figure 1 and Figure 2). The example dataset and pathway will be used to explain how both views can be used in Cytoscape.

When loaded as a pathway, the precise layout of elements is identical to its representation at WikiPathways. The graphical elements, like labels and shapes, are included in the model in Cytoscape. As a pathway diagram, the full representation of biological information is visually preserved, which is ideal for providing a meaningful context for data visualization. Figure 1 shows the Cardiac Hypertrophic Response pathway loaded as an annotated pathway in Cytoscape. The Entrez Gene identifiers in the pathway were mapped to Ensembl using another app called BridgeDb (http:// apps.cytoscape.org/apps/bridgedb) to match the identifiers used in the example dataset. The cardiac stem cell tissue development expression data can then be loaded, integrated and visualized on the pathway nodes (c.f. Introduction to Cytoscape tutorial, http:// opentutorials.cgl.ucsf.edu/index.php/Tutorial:Introduction\_to Cytoscapel_3.1-part2).
When loaded as a network, all graphical annotations are removed and redundant nodes in the pathway are merged into one unique node in the network. Groups and complex interactions are visualized as very small nodes and a forced directed layout is applied. As an abstracted network graph, the same molecular relationships in the pathways can be made available for network analysis and augmentation. Figure 2A shows the Cardiac Hypertrophic Response pathway loaded as a network in Cytoscape. This simple network structure enables researchers to use other Cytoscape features and apps to merge two pathways, apply different layouts to the network or extend the pathway, for example, with regulatory interactions (CyTargetLinker ${ }^{5}$, http://apps.cytoscape.org/apps/cytargetlinker). It also enables users to investigate the topology of the network, like calculating degree and betweenness of the nodes with Cytoscape's built-in NetworkAnalyzer tool to identify important hub nodes, see Figure 2B. Cytoscape also allows the visualization of experimental data in the network, as in Figure 2C which shows the cardiac stem cell tissue development expression data. There are several apps available for Cytoscape that provide methods that use experimental data to cluster nodes in the network (clusterMaker2, http://apps. cytoscape.org/apps/clustermaker2) or find subregions in the network affected by varying gene expression (jActiveModules, http://apps. cytoscape.org/apps/jactivemodules) as highlighted in Figure 2D.

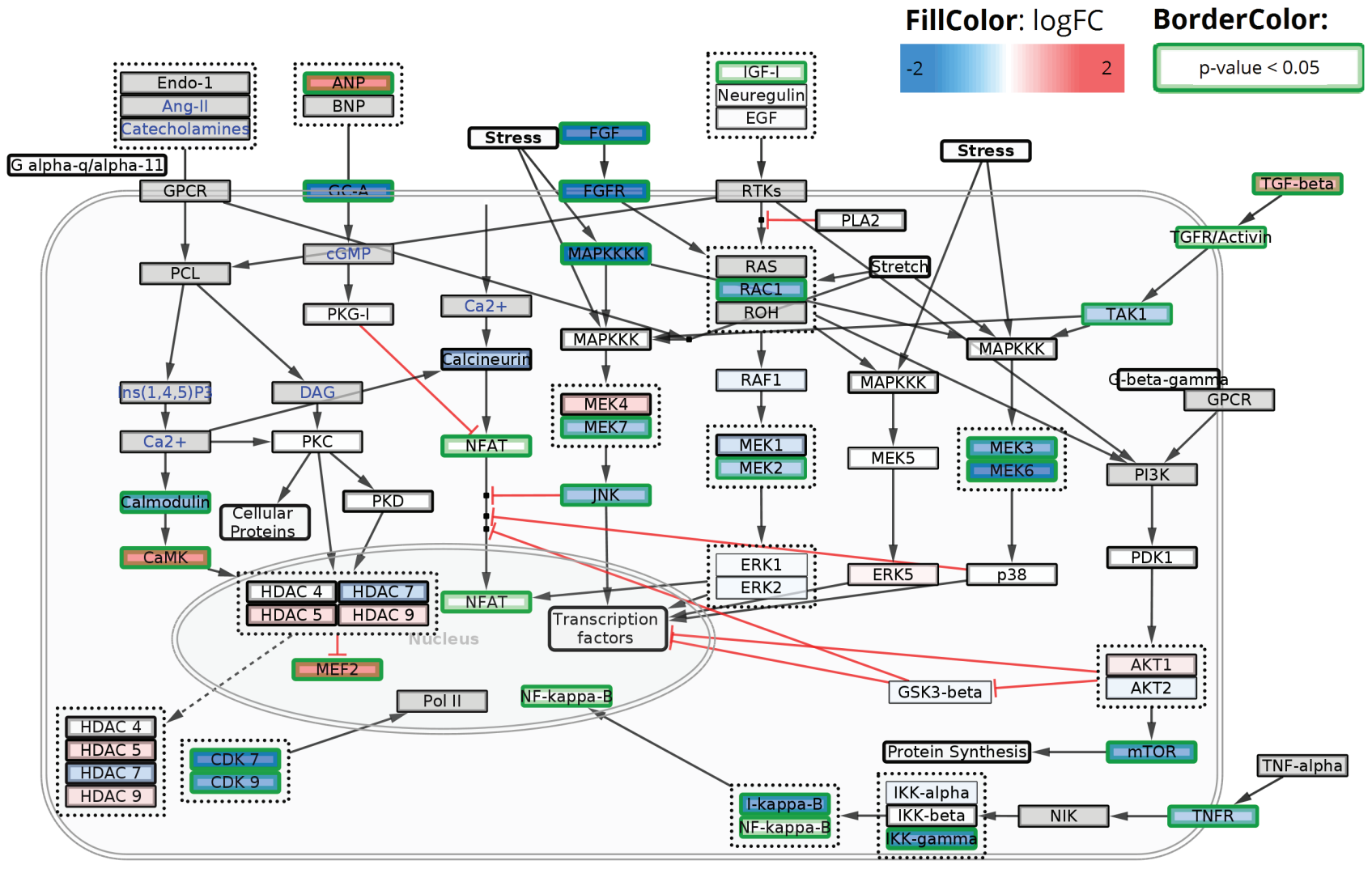

Figure 1. The Cardiac Hypertrophic Response pathway loaded as a pathway. LogFC values are visualized as node fill color with a color gradient from blue over white to red. Significant measurements (adjusted p-value $<0.05$ ) are highlighted with a green border color. Elements in the pathway without a measurement are colored grey. 

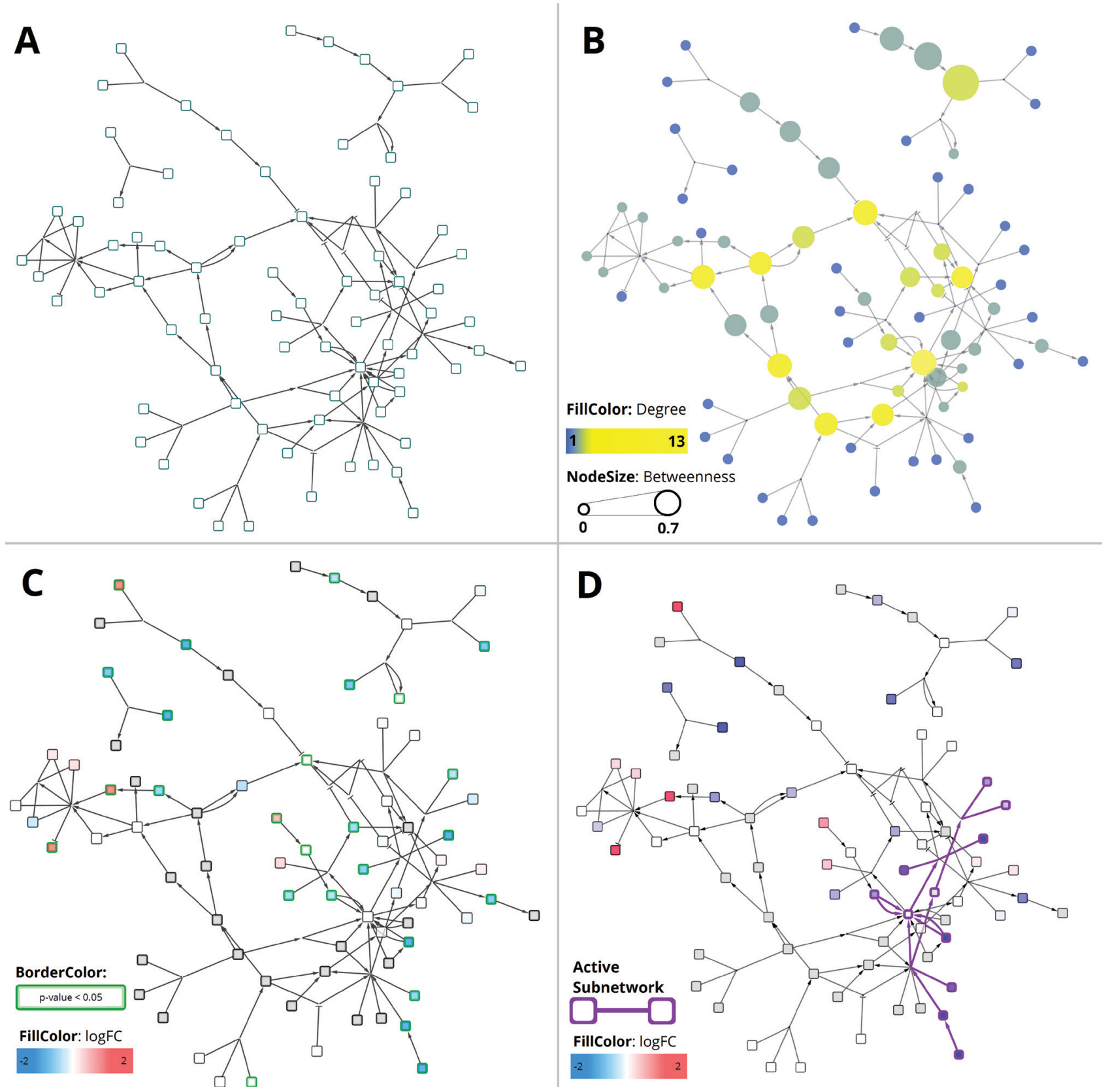

Figure 2. The Cardiac Hypertrophic Response pathway loaded as a network. (A) The simple network does not contain graphical annotations of the pathway. (B) NetworkAnalyzer was used to visualize node degree and betweenness of the nodes in the network to identify important hub nodes. (C) The logFC of the example dataset is visualized as node fill color with a gradient (blue over white to red) and the adjusted p-value $<0.05$ is highlighted with a green border color. (D) jActiveModules finds active subnetworks (highlighted in purple) that are affected by varying gene expression. 
Dataset 1. Dataset studying differentiation of cardiac stem cells.

This is a subset of an unpublished RNA-seq dataset containing measurements for all genes in the selected Cardiac Hypertrophy Response pathway comparing "time point 6 hrs vs. control". The dataset contains logFC, $\mathrm{p}$-value and adjusted $\mathrm{p}$-value measurements for every gene in the pathway.

Click here to access the data.

http://dx.doi.org/10.5256/f1000research.4254.d28415

\section{Conclusions}

In this paper we presented the WikiPathways app for Cytoscape, which allows the import of biological pathways as curated diagrams or as basic node-and-edge networks into Cytoscape. As shown in some examples, the app enables users to make full use of the pathway models by performing computational analyses and custom visualizations based on experimental data and network topology.

\section{Software availability}

Software available from the Cytoscape App Store: http://apps.cytoscape.org/apps/wikipathways

Latest source code: https://github.com/wikipathways/cytoscape-wikipathways-app

Source code as at the time of publication: https://github.com/F1000Research/cytoscape-wikipathways-app

Archived source code as at the time of publication: http://dx.doi. org/10.5281/zenodo. $10204^{7}$
License: Lesser GNU Public License 3.0: https://www.gnu.org/licenses/lgpl.html

\section{Data availability}

F1000Research: Dataset 1. Dataset studying differentiation of cardiac stem cells, 10.5256/f1000research.4254.d28415

\section{Author contributions}

MK, SL and ARP participated in the design of the described software. SL and MK implemented the WikiPathways app. MK, SL, CTE and ARP contributed to the development and writing of this article.

\section{Competing interests}

No competing interests were disclosed.

Grant information

We would like to acknowledge funding from NIGMS R01-GM100039 and NIDA U54-DA036134 (ARP, SL). This work was (co)financed by the Netherlands Consortium for Systems Biology (NCSB) which is part of the Netherlands Genomics Initiative/Netherlands Organisation for Scientific Research (MK, CTE).

The funders had no role in study design, data collection and analysis, decision to publish, or preparation of the manuscript.

Acknowledgments

We would like to thank Thomas Kelder for his work on the predecessor GPML plugin. Unpublished cardiac stem cell RNA-seq dataset courtesy of Dr. Bruce Conklin at the Gladstone Institutes. 


\section{Supplementary materials}

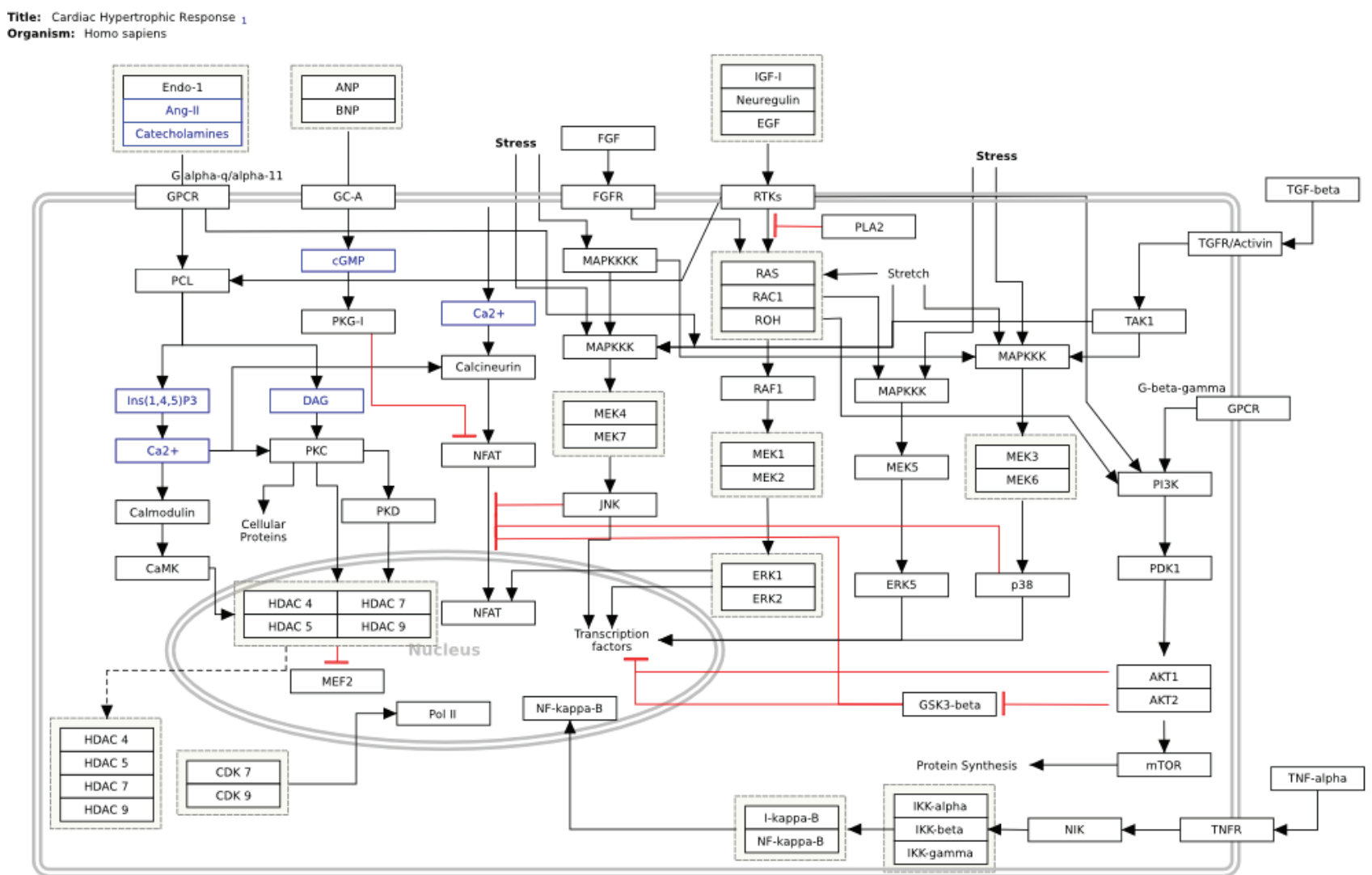

Supplementary Figure 1. Human Cardiac Hypertrophic Response pathway from WikiPathways used in the manuscript: http:// wikipathways.org/instance/WP2795_r74825. 


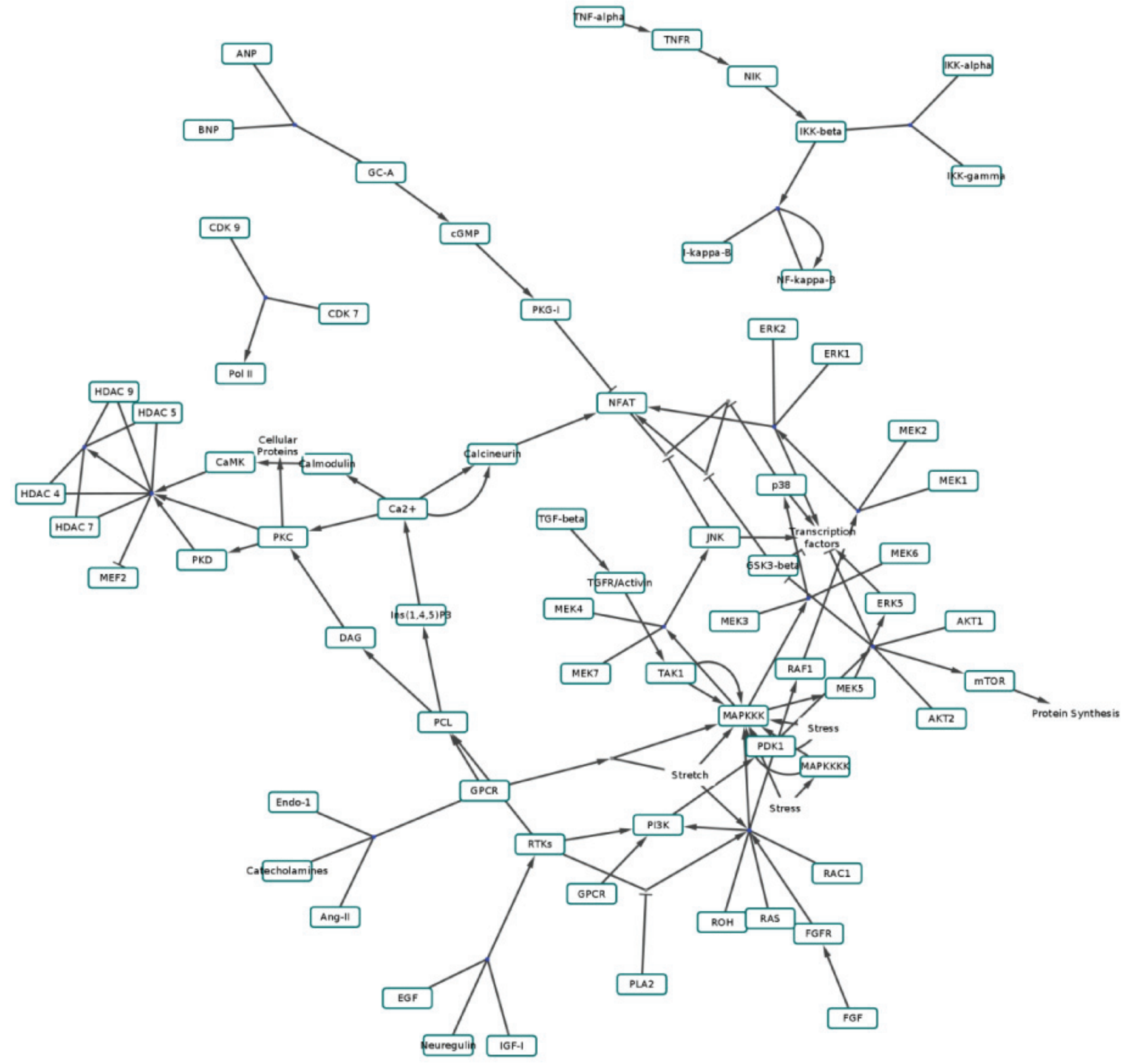

Supplementary Figure 2. Figure 2A in the WikiPathways App paper including all graphical details, e.g. Labels. 


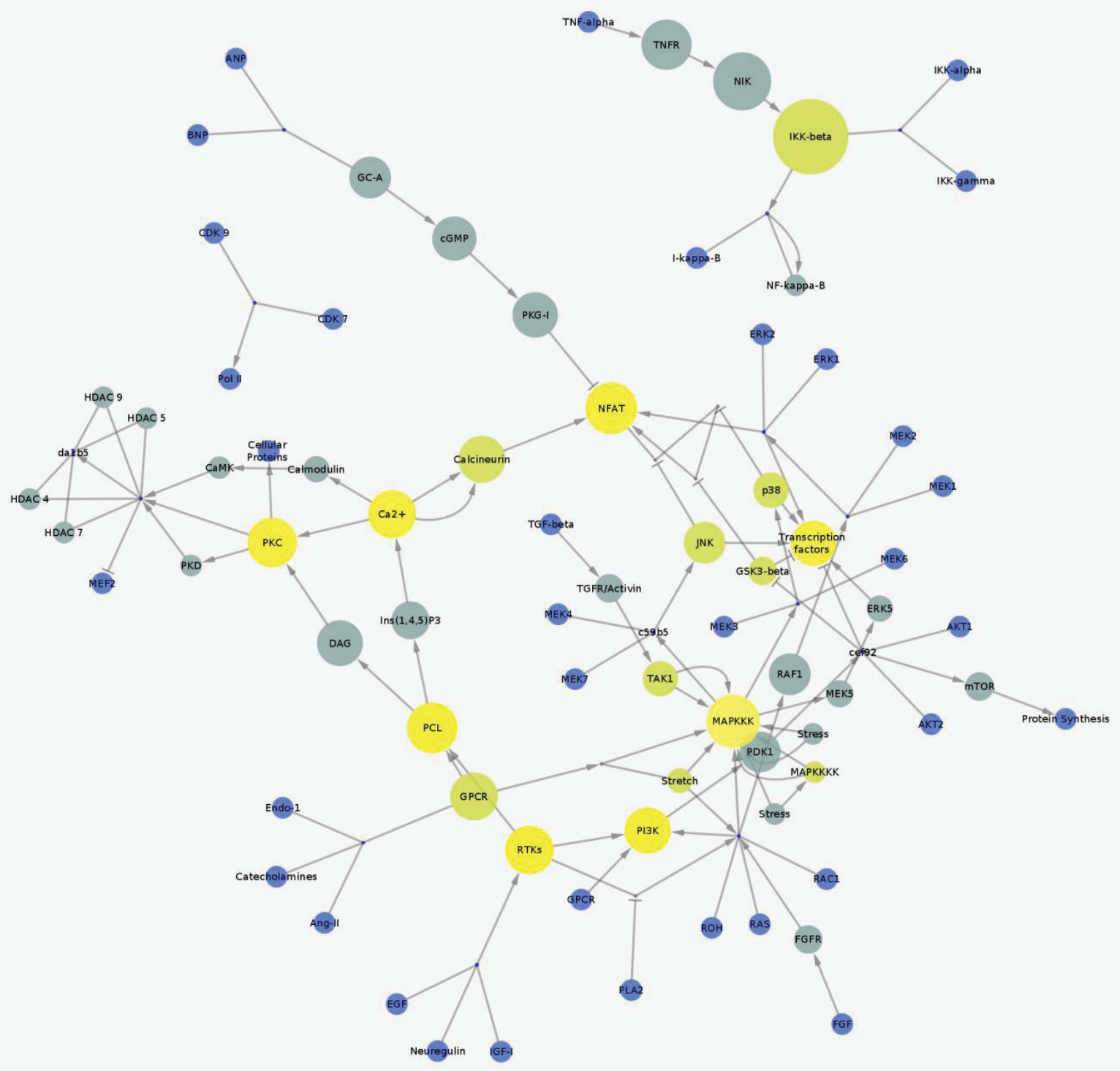

Supplementary Figure 3. Figure 2B in the WikiPathways App paper including all graphical details, e.g. Labels. 


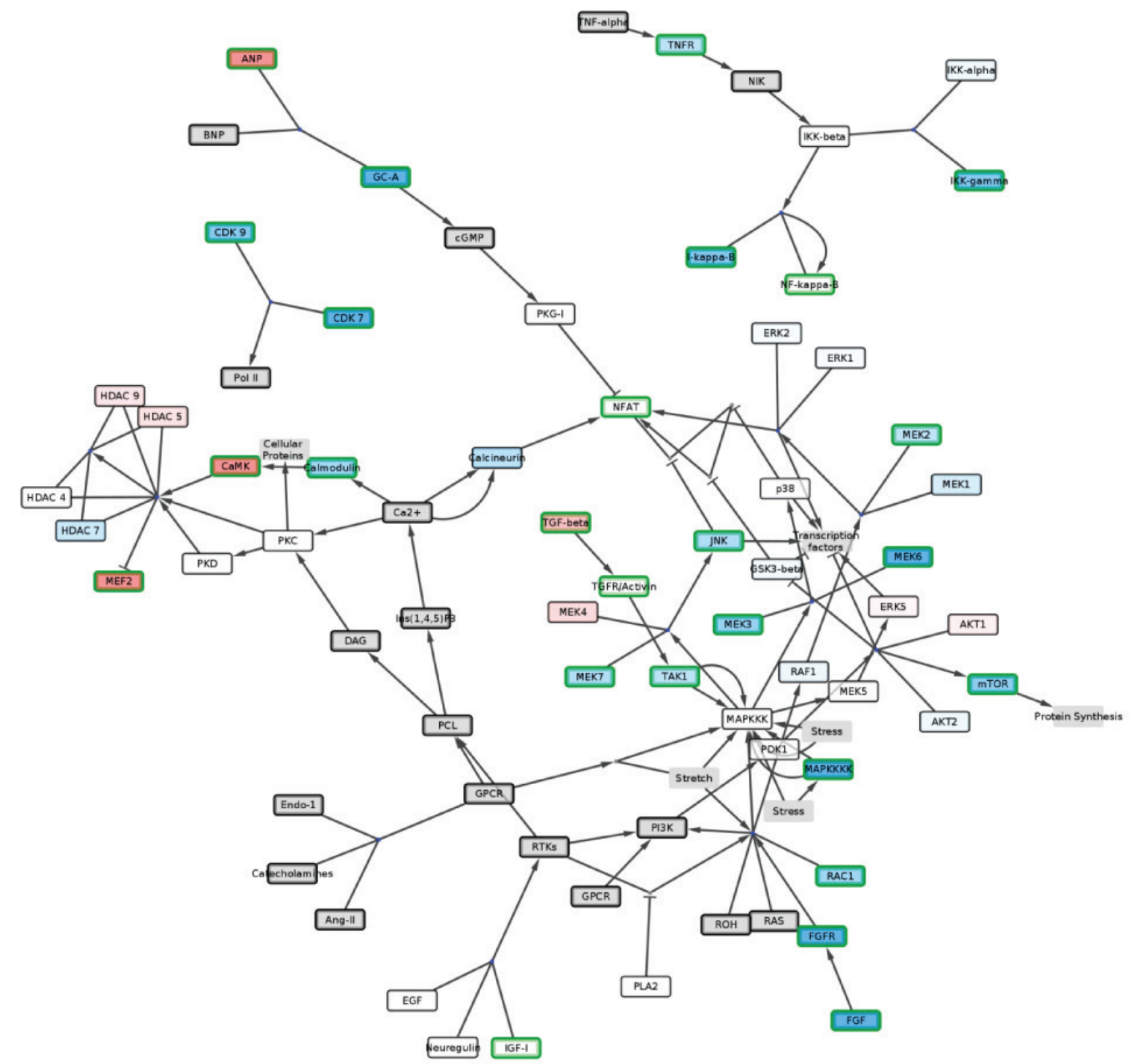

Supplementary Figure 4. Figure 2C in the WikiPathways App paper including all graphical details, e.g. Labels. 


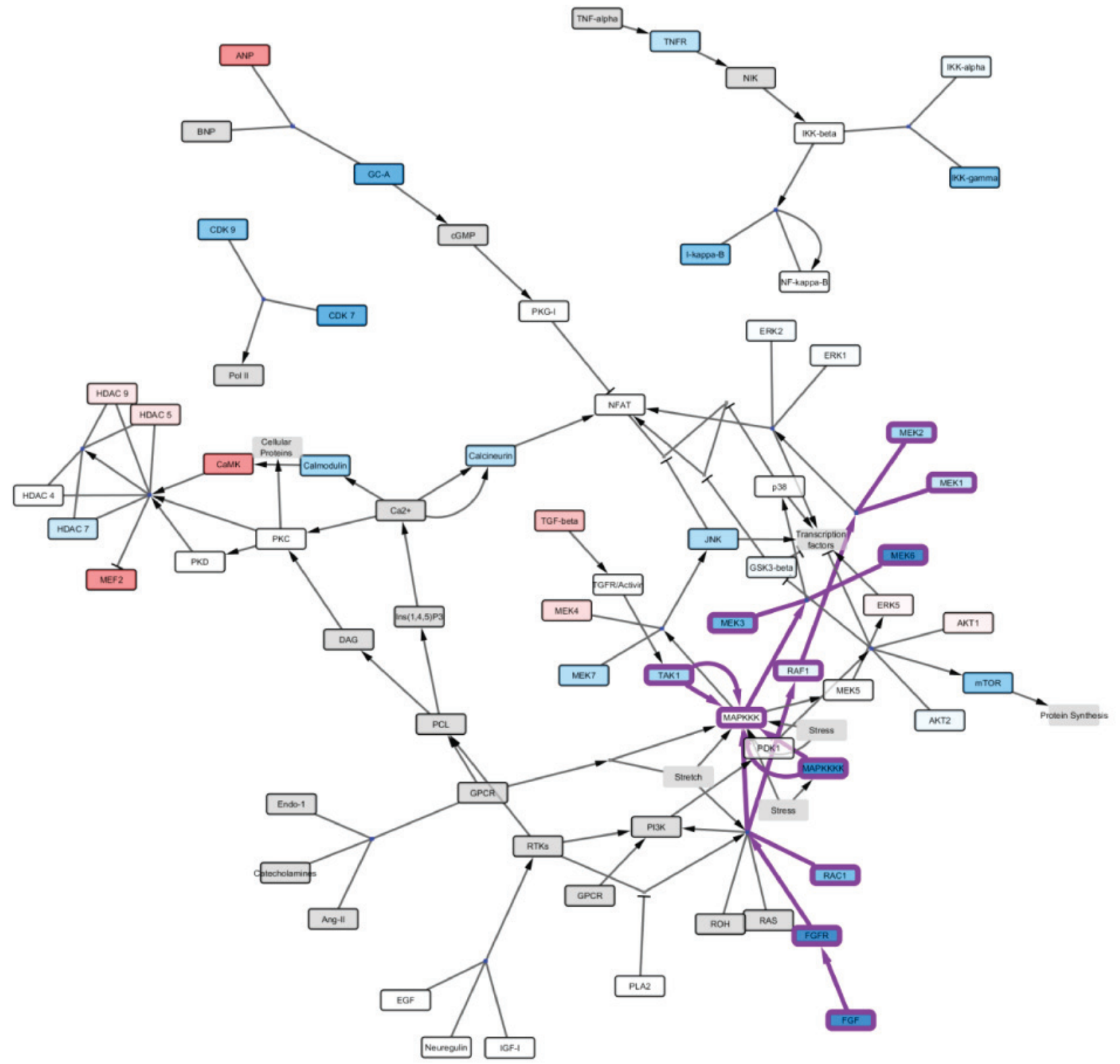

Supplementary Figure 5. Figure 2D in the WikiPathways App paper including all graphical details, e.g. Labels. 
1. Kelder T, van lersel MP, Hanspers $\mathrm{K}$, et al.: WikiPathways: building research communities on biological pathways. Nucleic Acids Res. 2012; 40(Database issue): D1301-D1307.

PubMed Abstract | Publisher Full Text | Free Full Text

2. Shannon P, Markiel A, Ozier O, et al:: Cytoscape: a software environment for integrated models of biomolecular interaction networks. Genome Res. 2003 13(11): 2498-2504.

PubMed Abstract | Publisher Full Text | Free Full Text

3. OSGi Alliance. Osgi service platform, release 3. IOS Press, Inc., 2003. Reference Source

4. van lersel MP, Kelder T, Pico AR, et al.: Presenting and exploring biological pathways with PathVisio. BMC Bioinformatics. 2008; 9(1): 399.

PubMed Abstract | Publisher Full Text | Free Full Text

5. Kutmon M, Kelder T, Mandaviya $\mathrm{P}$, et al.: CyTargetLinker: a cytoscape app to integrate regulatory interactions in network analysis. PLoS One. 2013; 8(12): e82160. PubMed Abstract | Publisher Full Text | Free Full Text

6. Kutmon M, Lotia S, Evelo CT, et al.: Dataset studying differentiation of cardiac stem cells. F1000Research. 2014.

Data Source

7. Kutmon M, Lotia S, Evelo CT, et al.: F1000Research/cytoscape-wikipathwaysapp. ZENODO. 2014

Data Source 


\section{Open Peer Review}

\section{Current Peer Review Status:}

\section{Version 1}

Reviewer Report 04 August 2014

https://doi.org/10.5256/f1000research.4555.r5523

(C) 2014 Goldstein T. This is an open access peer review report distributed under the terms of the Creative Commons Attribution License, which permits unrestricted use, distribution, and reproduction in any medium, provided the original work is properly cited.

\section{Ted Goldstein}

Department of Biomolecular Engineering, University of California Santa Cruz, Santa Cruz, CA, USA

You don't really make the point in the conclusions of the key findings of this excellent work which are:

1. It is now possible to do something quickly that before was tedious and therefore would not be done by anyone, except the most determined and experienced Cytoscape user.

2. It is now possible to do something that could not be done before because you needed to add new functionality.

I think it would be clearer that these are the main results if you add the following to the results section:

I suggest that you make a before and after video. The before video is the experience of using Cytoscape, manually downloading the data from Wikipathways, failing to see the the nodes because it does not have the DelayedVizProp class etc.

Then the after video shows the full experience.

The elapsed time of the before video is something like 10 minute while the after video is something like 1 or 2 minutes. This will clearly demonstrate the improvement in the user experience. Please report these time numbers in the text in the results section and possibly summarize the numbers in the abstract.

Of course, also post these videos to both F1000Research and Youtube as they represent the primary data of your study.

Competing Interests: No competing interests were disclosed.

I confirm that I have read this submission and believe that I have an appropriate level of expertise to confirm that it is of an acceptable scientific standard. 
Author Response 07 Aug 2014

Alexander Pico, Institute of Data Science and Biotechnology, Gladstone Institutes, San Francisco, CA, USA

Dear Dr. Goldstein,

Thank you for the enthusiastic review and suggestions. We have drafted a new conclusions paragraph to include in the next revision to the article, which should be posted very soon. Coincidentally, we were gearing up to prepare a series of introductory videos for WikiPathways, each under 10 minutes long. We will certainly have one covering the use of the WikiPathways app for Cytoscape and will link/post it here as well. Given limited time and resources, we might not get around to producing the "before" video, but we will describe the process for contrast. In fact, the "before" approach is not so clear. There are many routes to transforming an arbitrary XML like GPML into a input file for Cytoscape, ranging from the most basic SIF to the full XGMML formats. And for each format, one must make judgement calls and compromises, and ideally rely on someone with detailed knowledge of the source format. So, that approach could take anywhere from a day to a week of scripting depending on the desired accuracy and purpose. And, of course, it takes months of planning and programming (like we did) to get the general-purpose app result we arrived at.

Thanks again for highlighting these points and for your suggestions!

Competing Interests: No competing interests were disclosed.

Reviewer Report 02 July 2014

\section{https://doi.org/10.5256/f1000research.4555.r5286}

(C) 2014 Grove C. This is an open access peer review report distributed under the terms of the Creative Commons Attribution License, which permits unrestricted use, distribution, and reproduction in any medium, provided the original work is properly cited.

\section{Christian A Grove}

Division of Biology and Biological Engineering, California Institute of Technology, Pasadena, CA, USA

This paper is well written, clear and to-the-point. The title is appropriate and the abstract provides sufficient clarity and detail. The descriptions of the development, usage, and purpose of the WikiPathways App for Cytoscape are sufficiently detailed and informative for a bioinformatician such as myself. I cannot speak to the sufficiency of the detail of code and programming descriptions, although it seems reasonably complete. I've tested the App as the authors have described and found it to be easy to install and use with the set of descriptions and instructions provided on the source website and in this paper. All datasets and source code are easily 
obtainable with the links provided by the authors.

I have found one small typo in the network/pathway which I have manually changed at the WikiPathways.org site: the original network referred to the node of a gene product as "PCL", which according to the cited reference and the position in the network is surely meant to represent "Phospholipase C" which has the canonical abbreviation of "PLC", in the citation for the network and in numerous previous studies. Unless I have made this observation in error, I would recommend changing this in the network and associated figures.

Competing Interests: No competing interests were disclosed.

\section{I confirm that I have read this submission and believe that I have an appropriate level of expertise to confirm that it is of an acceptable scientific standard.}

Reader Comment 02 Jul 2014

Martina Summer-Kutmon,

Dear Dr. Grove,

Thank you for your very fast review. The typo you found is indeed a typo. We will update all figures accordingly. Thank you for immediately fixing it on WikiPathways yourself (http://wikipathways.org/instance/WP2795). This nicely highlights the efficiency of a wikibased pathway archive.

Competing Interests: No competing interests were disclosed.

The benefits of publishing with F1000Research:

- Your article is published within days, with no editorial bias

- You can publish traditional articles, null/negative results, case reports, data notes and more

- The peer review process is transparent and collaborative

- Your article is indexed in PubMed after passing peer review

- Dedicated customer support at every stage

For pre-submission enquiries, contact research@f1000.com 